\title{
Two-year outcomes of a pilot glaucoma suspect telemedicine monitoring program
}

This article was published in the following Dove Press journal:

Clinical Ophthalmology

\author{
Bobeck S Modjtahedi ${ }^{1,2}$ \\ Katherine Chu' \\ Tiffany Q Luong ${ }^{2,3}$ \\ Chunyi Hsu ${ }^{2,3}$ \\ Cynthia Mattox 4 \\ Paul P Lee ${ }^{5}$ \\ Mamdouh L Nakla ${ }^{6}$ \\ Donald S Fong ${ }^{1-3}$ \\ 'Department of Ophthalmology, \\ Southern California Permanente \\ Medical Group, Baldwin Park, CA, \\ USA; ${ }^{2}$ Eye Monitoring Center, Kaiser \\ Permanente Southern California, \\ Baldwin Park, CA, USA; ${ }^{3}$ Department \\ of Research and Evaluation, Southern \\ California Permanente Medical \\ Group, Pasadena, CA, USA; ${ }^{4} \mathrm{New}$ \\ England Eye Center, Tufts University \\ School of Medicine, Boston, MA, \\ USA; ${ }^{5}$ Department of Ophthalmology \\ and Visual Sciences, W.K. Kellogg \\ Eye Center, University of Michigan, \\ Ann Arbor, MI, USA; ${ }^{\circ}$ Department of \\ Ophthalmology, Southern California \\ Permanente Medical Group, South Bay \\ Medical Center, Harbor City, CA, USA
}

Correspondence: Bobeck S Modjtahedi Eye Monitoring Center, Kaiser Permanente Southern California, I0II Baldwin Park Boulevard, Baldwin Park, CA 91706, USA Email bobmodj@gmail.com
Purpose: The purpose of this study was to characterize a pilot program using e-health to monitor glaucoma suspects in a large integrated health system.

Methods: A retrospective chart review of patients enrolled in the first 2 years of a new glaucoma suspect telemedicine monitoring program was conducted. Patients were enrolled in the program after being diagnosed as glaucoma suspects in the regular clinic setting and were eligible for the program if they had better than 20/40 vision, intraocular pressure (IOP) $<25 \mathrm{mmHg}$, a normal baseline visual field, and an optical coherence tomography (OCT) retinal nerve fiber layer (RNFL) without clear evidence of glaucomatous optic nerve damage. Patients were followed annually thereafter with measurements of vision, IOP, and OCT RNFL, which were reviewed at a centralized telemedicine reading center. Patients were retained within the program unless there was evidence of disease progression, in which case they were referred to an ophthalmologist for further evaluation. The first 100 patients received a survey assessing their satisfaction with the program after their first visit. The number of patients who adhered to follow-up recommendations, who were referred to an ophthalmologist for additional evaluation, and who began on IOP-lowering medications was evaluated.

Results: A total of 225 patients were enrolled in this program. Of eligible patients, $97.3 \%$ attended their 1-year follow-up visit and $92.5 \%$ attended their 2-year follow-up visit. Over the course of 2 years, five patients were referred for further clinic evaluation due to concern for progressive RNFL loss, of which two were started on IOP-lowering medications. No patients were referred to the clinic for vision loss or elevated IOP. In all, $87 \%$ of patients said that they would be extremely or quite likely to recommend the program to a friend. More than $80 \%$ of patients said that the program was extremely or very helpful, convenient, and professional.

Conclusion: This novel telemedicine program for monitoring low-risk glaucoma suspects achieved high patient retention. Significant disease progression was rare with a few patients requiring referrals back to the clinic setting or initiation of IOP-lowering therapy. Telemedicine is a promising method to follow patients who are glaucoma suspects.

Keywords: e-health, glaucoma, optical coherence tomography, retinal nerve fiber layer, telemedicine, teleophthalmology

\section{Introduction}

Patients suspected of having glaucoma require regular monitoring with a combination of subjective and objective ophthalmic testing, which can be time consuming and costly for patients and practitioners, as well as society. The diagnosis of glaucoma relies on the presence of characteristic, acquired optic nerve morphology changes on clinical examination and/or retinal nerve fiber layer (RNFL) measurements on optical coherence tomography (OCT). Patients can have characteristic changes in their visual field testing based on the severity of their disease. Monitoring OCT RNFL has reshaped the 
management of glaucoma. OCT provides quantification of peripapillary RNFL thickness and a color-coded comparison of a patient's RNFL profile to an age-matched normative database. ${ }^{1}$ This color scheme highlights areas of probable RNFL defects in the overall contour of the standardized peripapillary circle scan, while overall average, quadrant, and clock hour quantifications of thickness are as clinically useful as numeric RNFL measurements. ${ }^{1}$ Global or sectoral RNFL thickness that is within $95 \%$ of normal is coded green, whereas RNFL thickness outside the $95 \%$ confidence interval (CI, but within the 99\% CI) is coded yellow, and values outside the $99 \%$ CI of the normal distribution are coded red. ${ }^{2-4}$ Figure 1 provides a sample of how the different sectors are divided within a typical report.

Glaucoma suspects are individuals who are at an increased risk of developing glaucoma due to a combination of clinical findings and risk factors including suspicious optic nerve appearance on examination or OCT, abnormal visual field, and/or elevated IOP. The monitoring of glaucoma suspects can represent a challenge especially when there are limited resources including patient access to ophthalmologists. Different ophthalmic societies, including the American Academy of Ophthalmology ${ }^{5}$ and the Canadian Glaucoma Society, ${ }^{6}$ provide detailed clinical recommendations for initial and periodic evaluations of glaucoma suspects with follow-up examinations recommended at least every 12-24 months, depending on individual risk.

Telemedicine is an increasingly popular method of delivering health care across multiple subspecialties. Interest in extending telemedicine into the routine screening and monitoring of ophthalmic pathologies has gained growing attention. $^{7}$ Although telemedicine screening programs for

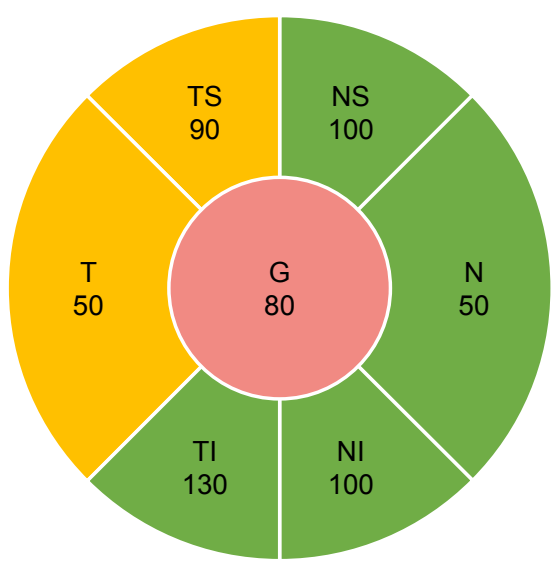

Figure I Example of OCT RNFL report.

Abbreviations: G, global; N, nasal; NI, nasal inferior; NS, nasal superior; OCT, optical coherence tomography; RNFL, retinal nerve fiber layer; T, temporal; TI, temporal inferior; TS, temporal superior. diabetic retinopathy and retinopathy of prematurity are well established, the use of telemedicine to monitor glaucoma suspects is an emerging area of interest.

The Kaiser Permanente Eye Monitoring Center (EMC) began a pilot project in 2013 to monitor low-risk glaucoma suspects. The initial 2-year experiences of this program are described herein to evaluate patient adherence/retention and to demonstrate a proof of concept.

\section{Methods}

The institutional review board (IRB) of Kaiser Permanente Southern California (KPSC) approved this study. The IRB does not require individual patient consents to be obtained for retrospective research where data are presented in an aggregate manner. KPSC is a group model health maintenance organization that provides prepaid health care to $\sim 4.5$ million members residing in Southern California. The medical group comprises $\sim 6,000$ physicians working from $>100$ medical offices and hospitals. Patients with Kaiser insurance are seen exclusively by physicians within the medical group, which has a single electronic medical record. The race and ethnic composition is similar to and representative of the population of Southern California.

Patients were referred to the EMC by an ophthalmologist or optometrist if they were diagnosed as a glaucoma suspect after a complete clinical evaluation including baseline visual fields, spectral domain OCT RNFL (Spectralis; Heidelberg Engineering, Dossenheim, Germany), assessment of family history/race, corneal pachymetry, and dilated fundus examination. Patients who were felt to be glaucoma suspects on clinic examination based on concerning optic nerve morphology, moderately elevated IOP (no greater than $25 \mathrm{mmHg}$ ), and/or areas of moderate RNFL loss (yellow color code in at least one sector or globally) on OCT were referred to the telemedicine program. Patients could be enrolled if they had 20/40 or better vision, an IOP $<25$ $\mathrm{mmHg}$, and a normal baseline visual field without evidence of glaucomatous optic nerve damage (and no sectors in the red color code) on OCT RNFL. Each patient's eligibility was evaluated by a glaucoma specialist and following approval they were enrolled in the program. These patients would receive an appointment at a local ophthalmology office where vision, IOP (via handheld applanation tonometer), and OCT RNFL were taken by a technician annually. These findings were forwarded to and screened by a trained technician at a centralized telemedicine reading center where they were compared to prior visits. Any patient with loss of two lines of Snellen visual acuity, increase in IOP by $\geq 5 \mathrm{mmHg}$, or a 
notable change in RNFL thickness on OCT globally in the temporal superior (TS) and/or temporal inferior (TI) quadrants (defined as $\geq 10 \mu \mathrm{m}$ reduction in the RNFL value or a change in the red color code) was then subject to additional review by a glaucoma specialist who evaluated the patient's clinical history and findings as well as imaging results. The quality of OCT scans based on signal strength, centration, and measurement markers was also assessed. Global, TS, and TI sectors were chosen as they have been shown to have the highest diagnostic accuracy. ${ }^{8}$ A $10 \mu \mathrm{m}$ decrease or a smaller change in thickness that resulted in a change of classification compared to the normative population database as outside the $99 \%$ CI of the normal distribution (ie, progression to the red color code) was chosen to reflect real-life variability in imaging results. ${ }^{2-4} \mathrm{~A}$ total of $10 \mu \mathrm{m}$ was chosen because the intervisit error rate for each quadrant was previously found to be $4 \mu \mathrm{m}$, and thus, $10 \mu \mathrm{m}$ represents a $>2$ SD variance. ${ }^{9}$ This threshold was the agreed upon standard set by glaucoma specialists at KPSC.

Patients who were deemed to have concerning features consistent with glaucomatous progression were referred to an ophthalmology clinic for further evaluation. Those patients who were not referred for ophthalmology clinic evaluation were retained in the program and received an appointment for a follow-up telemedicine visit in 1 year. The first 100 patients enrolled in this program received online surveys after their first visit where they were asked to grade how helpful, convenient, and professional the program was as well as if they would recommend the program to a friend. The purpose of this study was to assess patient adherence to recommended follow-up, retention within the program, patient referrals to an ophthalmologist for further evaluation, and progression to treatment requiring glaucoma.

\section{Results}

A total of 225 patients were enrolled in the program and had baseline OCT RNFL scans done. Of these initially enrolled patients, $87.1 \%$ completed their OCT RNFL scans at baseline, 1-year follow-up, and 2-year follow-up. Table 1 provides the baseline characteristics of the patients.

Between the baseline visit and 1-year follow-up, four patients changed insurance and were no longer eligible for the telemedicine program. Of eligible patients $(n=221), 97.3 \%$ of patients returned for evaluation and $2.7 \%$ of patients did not attend their prescribed appointment at the 1-year follow-up. Between years 1 and 2, five patients changed insurance plans, one patient was deceased, one patient followed up in ophthalmology clinic by their own accord, and one
Table I Baseline characteristics

All patients $(\mathbf{N}=\mathbf{2 2 5})$

\begin{tabular}{ll}
\hline Age in years & $58.1(14.2)$ \\
Mean (SD) & $18.5-87.9$ \\
Range & $142(63.1)$ \\
Females, $n$ (\%) & $558.45(35.09)$ \\
Mean baseline central corneal thickness (SD) \\
OD & $558.59(38.08)$ \\
OS & \\
Mean baseline mean deviation in dB (SD) & $-0.77(1.73)$ \\
OD & $-1.16(1.75)$ \\
OS & $14.82(2.97)$ \\
Mean baseline IOP in mmHg (SD) & $14.78(3.06)$ \\
OD ( $\mathrm{n}=223)$ & \\
OS ( $\mathrm{n}=223)$ & $98.56(10.46)$ \\
Mean global RNFL thickness in $\mu \mathrm{m}(\mathrm{SD})$ & $97.70(10.00)$ \\
OD & \\
OS & $143.68(20.54)$ \\
Mean TI OCT RNFL thickness in $\mu \mathrm{m}(\mathrm{SD})$ & $142.79(18.59)$ \\
OD & \\
OS & $136.64(18.87)$ \\
Mean TS OCT RNFL thickness in $\mu \mathrm{m}$ (SD) & $133.88(17.4 \mathrm{I})$ \\
OD &
\end{tabular}

Abbreviations: OCT, optical coherence tomography; RNFL, retinal nerve fiber layer; TI, temporal inferior; TS, temporal superior.

patient had been sent for clinic evaluation after their 1-year follow-up visit. There were 213 patients eligible for second year follow-up visit in the telemedicine program, of which 92.5\% attended their appointments and 7.5\% did not attend their prescribed follow-up. Figure 2 outlines patient movement within and outside the program.

There were 215 OCT RNFL measurements done at the year 1 follow-up visit and 197 at the year 2 follow-up visit. There was a minimal reduction in OCT RNFL thickness (Figure 3).

Five patients $(2.3 \%)$ had notable global RNFL loss ( $\geq 10 \mu \mathrm{m}$ reduction in RNFL value or a change in the red color code) at 1-year follow-up: in four patients OCT RNFL measures remained in the green color code and one patient progressed to the red color code in the global and TS sectors (see below) and was referred by the EMC for clinical evaluation. In all, 20 (9.3\%) patients had notable TS and/or TI RNFL loss at year 1 follow-up: 16 had $\geq 10 \mu \mathrm{m}$ loss in RNFL but no change in color map, two patients progressed from green color codes to yellow sectors, and two patients progressed from green to red color codes in the TS sector (one patient also progressed from green to red in global RNFL as noted earlier and the other patient also progressed from green to yellow in the TI sector). The latter of these two patients personally elected to go for clinical evaluation 


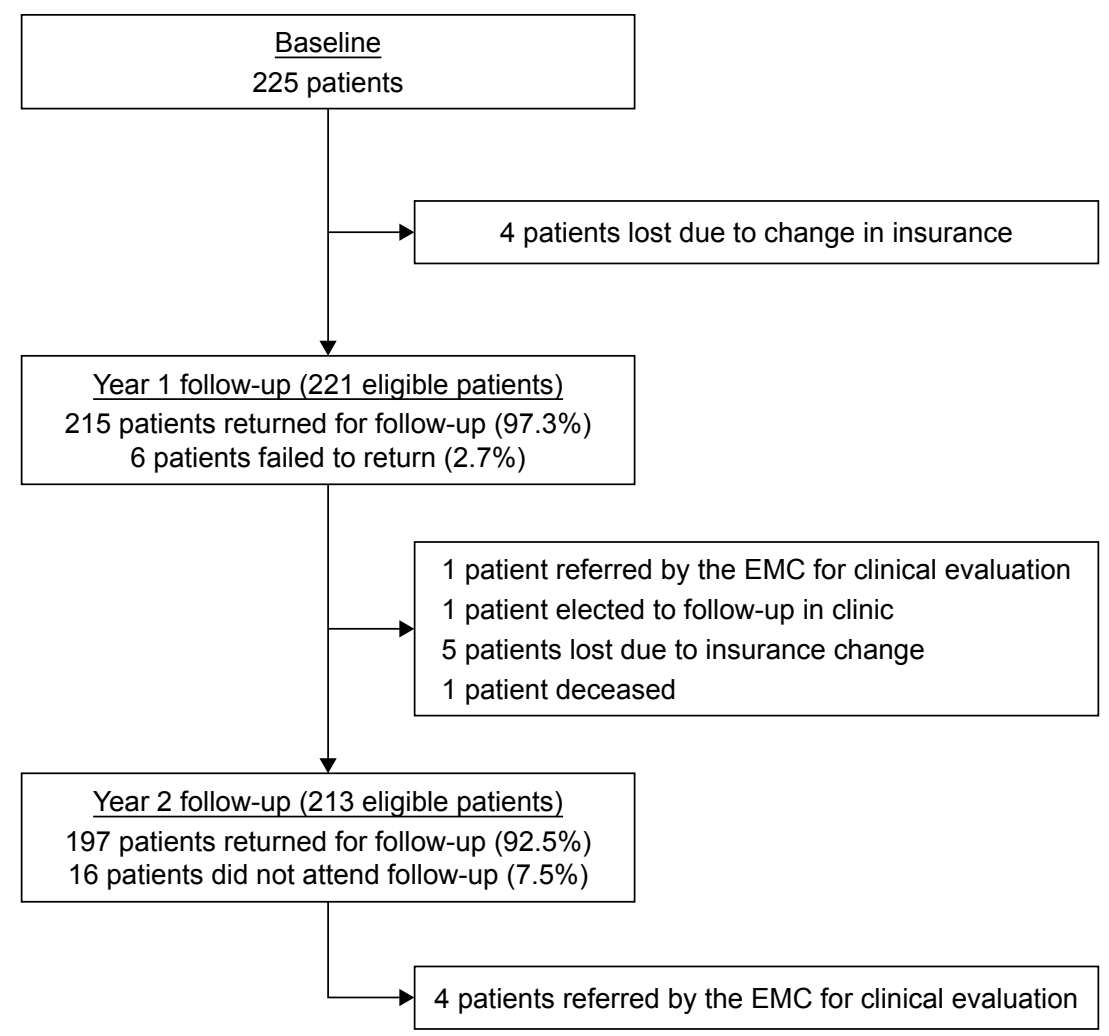

Figure 2 Patient flow.

Abbreviation: EMC, Eye Monitoring Center.

instead of returning for repeat testing in the reading center, where she was deemed to be stable and not started on glaucoma therapy.

Three (1.5\%) patients at 2-year follow-up had notable global RNFL loss, none of whom were sent for ophthalmology evaluation: one patient remained in the green color code in all sectors, one patient had progression to the yellow color code in the TI sector but remained in the green color code globally, and one patient had progression to the red color code but was not referred for clinical evaluation after review by a glaucoma specialist because this change was due to an imaging error. In all, 35 (17.8\%) patients had notable TS and/or TI loss at their year 2 follow-up: 26 had $\geq 10 \mu \mathrm{m}$ loss in RNFL but no change in color map, eight progressed from green to yellow sectors (four sent for clinical evaluation), and one patient developed a new red sector but was not sent to ophthalmology after review of his scans by a glaucoma specialist because the change was due to an error in the measurement bars on the scan not correctly tracking the RNFL.

Five patients over the course of 2 years met criteria for clinic referral. One patient was sent for clinic evaluation after their 1-year follow-up visit $(0.47 \%$ of evaluated patients) where it was determined that the screening center OCT was inaccurate and that the patient was in fact stable.
No treatment was subsequently started. Following the year 2 visit, four patients $(0.020 \%$ of evaluated patients $)$ were sent for clinical evaluation of which two were prescribed glaucoma medication, one was observed, and one failed to attend his appointment.

Figure 4 provides a summary of survey responses from the first 100 patients after their first visit. More than $80 \%$ of patients found the program extremely or very helpful, convenient, and professional. In all, $87 \%$ of patients were extremely or quite likely to recommend the program to a friend.

\section{Discussion}

Telemedicine programs are a promising means to alleviate strain on the health care system in the face of growing demand for health care in general and ophthalmic care specifically as societies age. Early stages of glaucoma are ideal for telemedicine programs because glaucoma is a slowly progressive disease where small incremental progression is less likely to be visually significant in the early phases.

Novel programs that rely on the use of specially trained optometrists in a defined framework have been developed to alleviate some of the health care burden placed on ophthalmologists and glaucoma specialists. ${ }^{10,11} \mathrm{~A}$ program utilizing "virtual visits" in the UK found a low rate of 
A

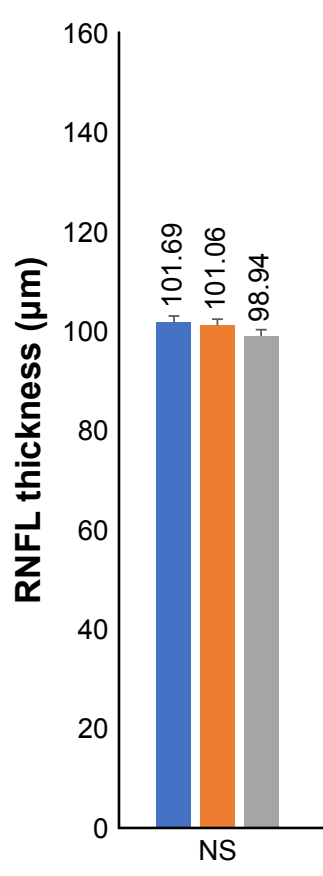

Mean OCT measurements for the right eye

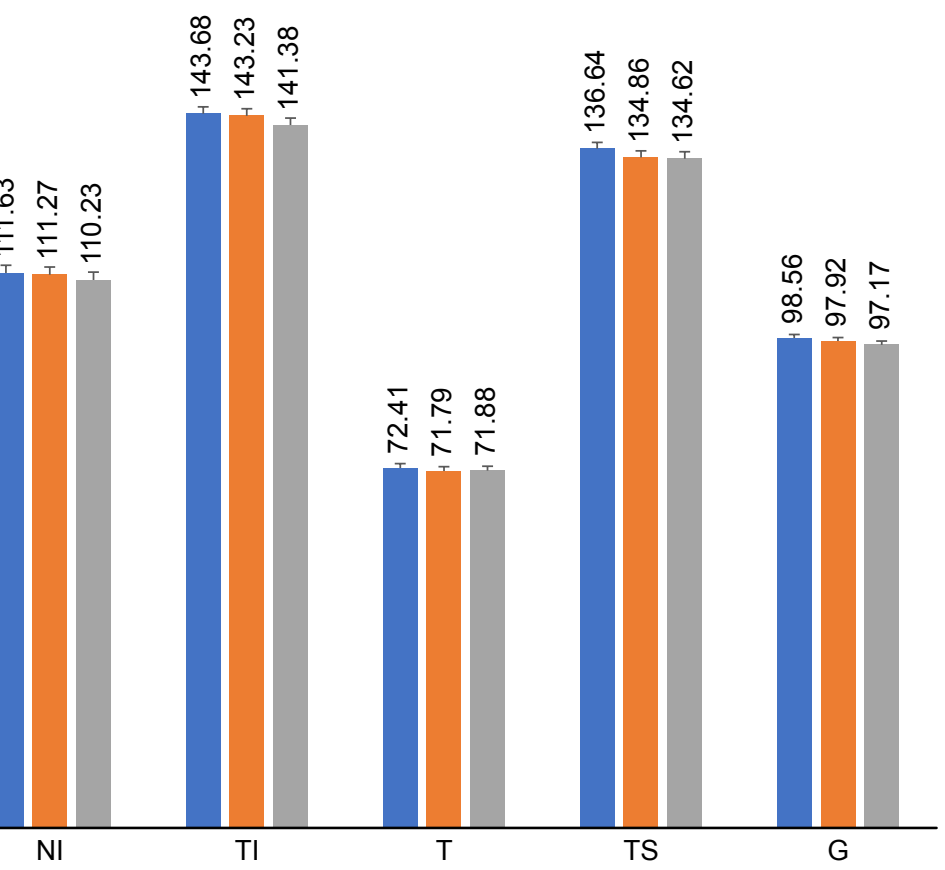

B



Figure 3 OCT RNFL measurements over 2 years.

Notes: (A) Right eye. (B) Left eye.

Abbreviations: G, global; N, nasal; NI, nasal inferior; NS, nasal superior; OCT, optical coherence tomography; RNFL, retinal nerve fiber layer; T, temporal; TI, temporal inferior; TS, temporal superior.

adverse misclassification when comparing patients evaluated remotely and those evaluated with a face-to-face visit. ${ }^{12}$ A technician-delivered glaucoma referral triage system, with glaucoma specialist providing virtual review of data at a later time after acquisition, significantly reduced onward glaucoma referrals and had a positive predictive value of onward glaucoma referrals of $84 \%$ and a negative predictive value of $96 \% .{ }^{13}$ Tele-glaucoma programs have been found to 

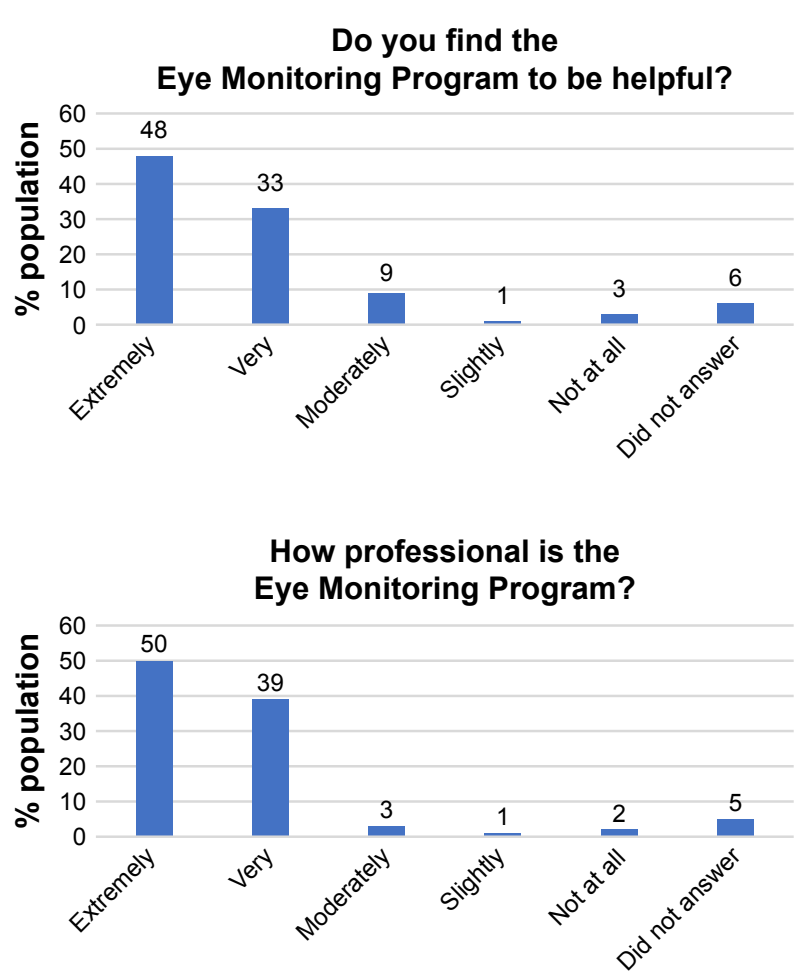

Figure 4 Survey responses of the first 100 patients enrolled in the program.

be less sensitive and more specific than in-person screening, while also being cost-effective. ${ }^{14}$

A significant limitation of prior studies of glaucoma telemedicine programs is that they often do not have longitudinal follow-up. ${ }^{14}$ In contrast, the presented pilot study provides follow-up over 2 years. Adherence to recommended follow-up in screening telemedicine programs, as well as in regular clinic settings, represents a significant problem in glaucoma care. ${ }^{15}$ There was a high patient adherence to recommended follow-up and high patient retention within the $\mathrm{EMC}$ as $97.5 \%$ and $92.5 \%$ of eligible patients came to their year 1 and year 2 follow-up visits, respectively. Unlike other telemedicine programs that have focused on screening for glaucoma, this program took patients who already received a baseline clinical evaluation and provided monitoring. In traditional screening models, patients receive imaging that is reviewed at a later time by a technician or a clinician. If pathology is detected, then the patient has to be contacted and told to return for further evaluation. An innate limitation to the traditional screening model is that it can be difficult to contact some patients and these patients may go unaware that they have a sight-threatening condition. The model proposed herein has several advantages, including the ability of the patients to receive an in-depth in-person discussion of their disease at the time of diagnosis where the importance of adherence to
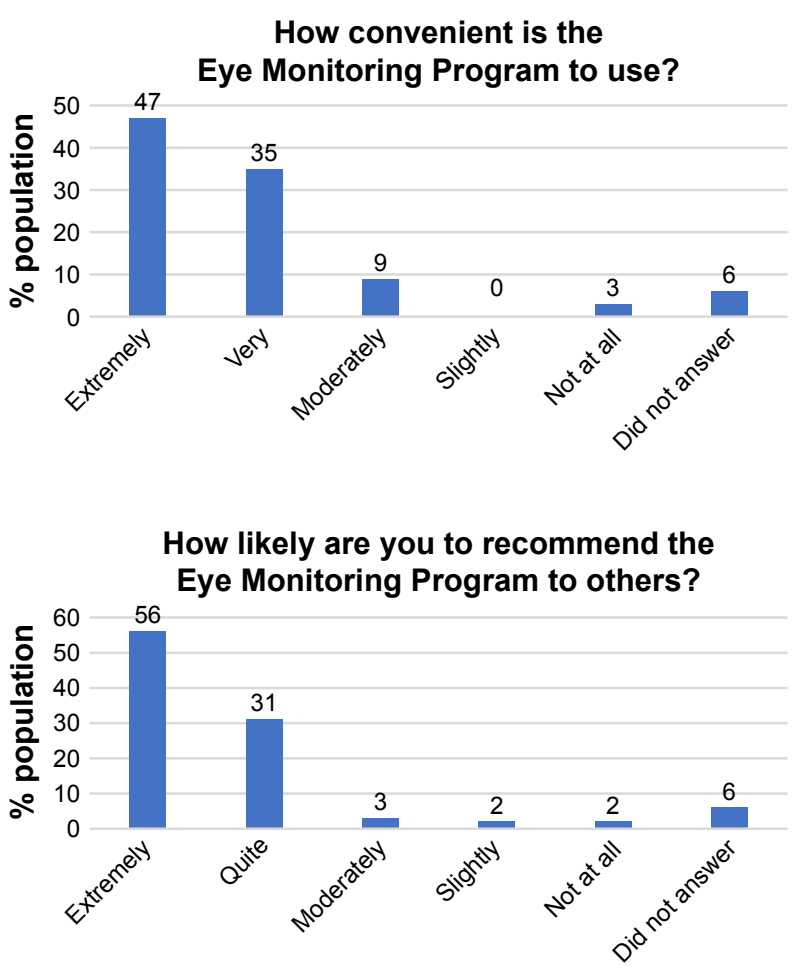

follow-up can be emphasized. Telemedicine is then used to monitor, as opposed to diagnose, patients who have already bought in to the importance of the telemedicine program. Additionally, patients in this program came to local eye clinics to have their testing done, with results sent remotely to the centralized reading center, which created ease of access for most patients. The adherence to follow-up recommendations was substantially higher in our study compared to others (including traditional clinic follow-ups), which are typically about $60 \% .{ }^{15}$ Factors such as socioeconomic status, education level, and insurance/financial considerations make comparisons between studies difficult; however, we hypothesize that the initial clinic evaluation where patients received education about their condition and telemedicine, combined with the ease of access created by being part of the EMC, resulted in this high adherence to follow-ups. Patient satisfaction was high within this program with $87 \%$ of patients saying that they would be extremely or quite likely to recommend the program to a friend and $>80 \%$ of patients finding the program extremely or very helpful, convenient, and professional. A randomized trial comparing traditional clinic follow-up and telemedicine programs would further help define differences between these two different practice models.

The purpose of this study was to evaluate the initial performance of a new glaucoma suspect telemedicine 
monitoring program. Additional studies will be necessary to determine the longer term success of these programs, especially given the typically slow disease progression of glaucoma suspects. Only five patients required in-person clinical evaluation due to possible disease progression noted by the EMC, and of them, only two ultimately required the initiation of glaucoma medications. Thus, the EMC can be reasonably concluded to have reduced the clinic burden that would have been created by the remaining patients who did not need to be seen in clinic. The low rate of patients who required glaucoma treatment $(0.0089 \%)$ was comparable to the risk of developing primary open-angle glaucoma in the observation group of the Ocular Hypertension Treatment Study at 24 months $(0.012 \%) .{ }^{16}$ In addition to alleviating the strain on clinical time and resources, this model also benefits patients by providing a less time-consuming experience. Only one patient self disenrolled from the EMC in favor of following up in a regular clinic setting. One limitation of the program described herein is that visual fields were not taken on follow-up; however, serial OCT RNFLs represent a simple strategy to follow patients since RNFL loss typically pre-dates visual field loss and significant RNFL reduction is necessary before patients have clinical manifestations. ${ }^{4,17}$ There is a decay of RNFL thickness with age in normal individuals, especially after 50 years. ${ }^{18}$ Glaucoma suspects who develop visual field defects exhibit a rate of global RNFL loss generally at least twice as fast as those who do not develop visual field defects. ${ }^{19}$ Patients within this program were at low risk (as seen in the results), and our results may not be generalizable to other glaucoma populations. Further effort should be made to refine the clinical parameters for enrollment and evaluation within such programs. Future investigations should explore expanding these telemedicine services to higher risk populations to increase the clinical impact of these programs.

\section{Disclosure}

DS Fong has the following disclosure outside of the submitted manuscript: grants from Allergan, ThromboGenics, and Nightstar. PP Lee has the following disclosures outside the submitted work: consultant for Alcon and equity owner in Pfizer, GSK, Merck, and Medco Health Solutions. C Mattox has the following disclosures outside of the submitted manuscript: grants and personal fees from Alcon and Allergan and personal fees from Aerie Pharmaceuticals, New World
Medical, and Novartis. The authors report no other conflicts of interest in this work.

\section{References}

1. Kim CY, Jung JW, Lee SY, Kim NR. Agreement of retinal nerve fiber layer color codes between Stratus and Cirrus OCT according to glaucoma severity. Invest Ophthalmol Vis Sci. 2012;53(6):3193-3200.

2. Heidelberg Engineering; Dossenheim, Germany. OCT Acquisition Window-Overview. Spectralis OCT QuickGuide. (Software Version 4.0); 2008.

3. Bendschneider D, Tornow RP, Horn FK, et al. Retinal nerve fiber layer thickness in normals measured by spectral domain OCT. J Glaucoma. 2010;19(7):475-482.

4. Wu H, de Boer JF, Chen TC. Diagnostic capability of spectral-domain optical coherence tomography for glaucoma. Am J Ophthalmol. 2012;153(5):815e812-826e812.

5. Prum BE, Lim MC, Mansberger SL, et al. Primary Open-Angle Glaucoma Suspect Preferred Practice Pattern $\left({ }^{\circledR}\right)$ Guidelines. Ophthalmology. 2016;123(1):P112-P151.

6. Canadian Glaucoma Society Committee on Interprofessional Collaboration in Glaucoma Care. Model of interprofessional collaboration in the care of glaucoma patients and glaucoma suspects. Can J Ophthalmol. 2011;46(6 Suppl):S1-S21.

7. Rathi S, Tsui E, Mehta N, Zahid S, Schuman JS. The Current State of Teleophthalmology in the United States. Ophthalmology. 2017; 124(12):1729-1734.

8. Lisboa R, Leite MT, Zangwill LM, Tafreshi A, Weinreb RN, Medeiros FA. Diagnosing preperimetric glaucoma with spectral domain optical coherence tomography. Ophthalmology. 2012;119(11):2261-2269.

9. Ghasia FF, El-Dairi M, Freedman SF, Rajani A, Asrani S. Reproducibility of spectral-domain optical coherence tomography measurements in adult and pediatric glaucoma. J Glaucoma. 2015;24(1):55-63.

10. Henson DB, Spencer AF, Harper R, Cadman EJ. Community refinement of glaucoma referrals. Eye. 2003;17(1):21-26.

11. Devarajan N, Williams GS, Hopes M, O'Sullivan D, Jones D. The Carmarthenshire Glaucoma Referral Refinement Scheme, a safe and efficient screening service. Eye. 2011;25(1):43-49.

12. Clarke J, Puertas R, Kotecha A, Foster PJ, Barton K. Virtual clinics in glaucoma care: face-to-face versus remote decision-making. $\mathrm{Br} \mathrm{J}$ Ophthalmol. 2017;101(7):892-895.

13. Kotecha A, Brookes J, Foster PJ. A technician-delivered "virtual clinic" for triaging low-risk glaucoma referrals. Eye. 2017;31(6):899-905.

14. Thomas SM, Jeyaraman MM, Jeyaraman M, et al. The effectiveness of teleglaucoma versus in-patient examination for glaucoma screening: a systematic review and meta-analysis. PLoS One. 2014;9(12):e113779.

15. Hark LA, Katz LJ, Myers JS, et al. Philadelphia Telemedicine Glaucoma Detection and Follow-up Study: Methods and Screening Results. Am J Ophthalmol. 2017;181:114-124.

16. Kass MA, Heuer DK, Higginbotham EJ, et al. The Ocular Hypertension Treatment Study: a randomized trial determines that topical ocular hypotensive medication delays or prevents the onset of primary openangle glaucoma. Arch Ophthalmol. 2002;120(6):701-713.

17. Wu H, de Boer JF, Chen L, Chen TC. Correlation of localized glaucomatous visual field defects and spectral domain optical coherence tomography retinal nerve fiber layer thinning using a modified structurefunction map for OCT. Eye. 2015;29(4):525-533.

18. Parikh RS, Parikh SR, Sekhar GC, Prabakaran S, Babu JG, Thomas R. Normal age-related decay of retinal nerve fiber layer thickness. Ophthalmology. 2007;114(5):921-926.

19. Miki A, Medeiros FA, Weinreb RN, et al. Rates of retinal nerve fiber layer thinning in glaucoma suspect eyes. Ophthalmology. 2014;121(7): $1350-1358$. 


\section{Publish your work in this journal}

Clinical Ophthalmology is an international, peer-reviewed journal covering all subspecialties within ophthalmology. Key topics include: Optometry; Visual science; Pharmacology and drug therapy in eye diseases; Basic Sciences; Primary and Secondary eye care; Patient Safety and Quality of Care Improvements. This journal is indexed on

Submit your manuscript here: http://www.dovepress.com/clinical-ophthalmology-journal
PubMed Central and CAS, and is the official journal of The Society of Clinical Ophthalmology (SCO). The manuscript management system is completely online and includes a very quick and fair peer-review system, which is all easy to use. Visit http://www.dovepress.com/ testimonials.php to read real quotes from published authors. 